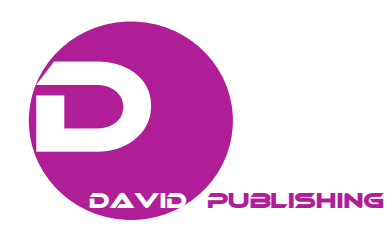

\title{
Recent Data on Birds of Kinshasa in Democratic Republic of Congo
}

\author{
Julien Kumanenge Punga ${ }^{1}$ and Séraphin Ndey Bibuya Ifuta ${ }^{2}$ \\ 1. Department of Biology, Faculty of Science, University of Kinshasa, P.O. Box 190, Kinshasa, Democratic Republic of Congo \\ 2. Department of Biology, Teaching Higher Institute of Gombe, P.O. Box 3580, Gombe, Kinshasa, Democratic Republic of Congo
}

\begin{abstract}
The study aimed at understanding the current avifauna characteristics, like composition, species diversity and evolution, in the city of Kinshasa. The study was conducted from 2006 to 2014, using observation, photography and Japanese nets. Results of the study indicate that there are 131 species of birds, which represents 40 families and 16 orders. Avifauna of Kinshasa represents $11 \%$ of species of the all country. Among those species, 12 are new. Passerines are the most, representing 86 species and 21 families, and are the most diversified. Few species have extended their geographical distribution and some are migratory. Overtime, avian fauna of Kinshasa region has undergone a lot of changes in its composition and diversity. Horizontal extension of the city associated with the consecutive various changes of the habitats seems to be the principal factors which modulate those characteristics. However, the study found that the majority of these species were under precarious statute of conservation.
\end{abstract}

Key words: Birds, specific diversity, Kinshasa, Democratic Republic of Congo.

\section{Introduction}

\subsection{Goals of the Study}

Birds have been the subject of several studies in the Democratic Republic of Congo (DRC), formerly called Belgian Congo and after Zaire [1-6] before his actual name. The town of Kinshasa is among localities, where various collections of data were carried out but its birds are mixed with those of the whole country.

The first study of the birds of Léopoldville (Kinshasa) was done about 50 years ago; even though it was enriched thereafter [7], only few data on the birds of Kinshasa still are available.

Kinshasa is a city that has undergone a lot changes, so was its habitat. The present study, which is a follow-up of a previous study that was conducted 13 years ago [8], intended to show specificity of the avian fauna of Kinshasa, its diversity as well as its current main characteristics.

Corresponding author: Julien Kumanenge Punga, professor, research field: animal behavioral ecology. E-mail: pungaku@yahoo.fr; julienpungaku@gmail.com.

\subsection{Habitat}

Kinshasa, formerly called Leopoldville, was founded in December 1881 [9] and had a population of 5,000 inhabitants in 1884 , living on 115 ha with a density of 43.5 inhabitants/ha [10]. Kinshasa become the political capital of the country and acquired the status of a province. Currently, the city extends on an area of $9,984.5 \mathrm{~km}^{2}$ [10]. Its population was estimated at more than 8 million inhabitants in 2010 [11] and very close to 10 million today. It is a sprawling town located in the Southwest of the country with Southern latitude $4^{\circ}-5^{\circ}$ and Eastern longitude $15^{\circ}-16^{\circ} 30^{\prime}$ [12]. Its climate is of tropical type AW4 according to the classification of Köppen [13].

From geomorphologic standpoint, Kinshasa presents varied aspects: plain zone with low terraces along the shores of the Congo River. However, when moving away from the Congo River, Kinshasa also presents a succession of hills from lowlands to the Bateke Plateau, which prolongs to the one of Kwango. 
Considering its area, the city covers a mosaic of habitats, with a various and diversified flora settlement which has not entirely been studied [14-19]. Downtown area is particularly occupied by fruit trees, among which mango (Manguifera indica), avocado (Percea Americana) and palm oil (Elaeis guineensis) are the most representative. The urban districts constitute its own field with several fruit trees, of which it is necessary to include papaya (Carica papaya), safoutier (Dacryoides edulis), red lawyer (Syzigium cumini), various vegetables, ornamental plants like blazing (Deonyx regia) and wenge (Millettia laurentii), hedge's plants like Chromolaena odorata, Lantana camara and Tithonia diversifolia, and many ruderal plants. In peripheral rural, Panicum maximum, Hyparhenia spp. and Pennisetum purpureum generally dominate. It has to be also noticed the presence of forest galleries and grassy mosaic formations. This entire habitat offers an environment fertile to bird's life associated or not to man's presence.

\section{Materials and Methods}

The authors combined direct observation of birds by the binoculars with Japanese nets capture. The authors had explored various locations, like forest, gardens, dwelling house including a vegetation able to attract birds, along the rivers, etc.. In some cases, the authors also used a Canon IXUS 4 camera and an Olympus camera for pictures. For the identification of species, many works were used [20-27]. Phylogenetic classification $[28,29]$ has brought many changes at the level of the orders, families, genera and species of birds. In this work, recent classification [30-32] was used.

Referring to prior data [7], it seemed that many species had changed names or systematic position or both. Thus, the authors brought up to date names of birds and their systematic positions as indicated on Table 1 before generating a general list of the known species of Kinshasa.

\section{Results}

\subsection{Composition}

Current avian fauna of Kinshasa has 16 orders, 41 families and 131 species (Table 2). Sparrows are the most with 21 families and 86 species. The majority of these species mainly live in open habitats; few are forest birds like Bulbul, few others are aquatic birds particularly Herons and the last are ubiquist birds like Columbidae. Some pictures of those birds are in Fig. 1.

\subsection{Specific Diversity}

Among the above species listed, there is an unequal distribution of species between orders and families. Passerines are the most representative in diversity of species and they include 86 species which is $66 \%$ of birds. In second position, there are two ordersPiciformes and Coraciiformes. Each one of them has two families with nine species for the first and eight species for the latter, respectively. All other orders have one or two families with one or three species.

\subsection{Geographical Repartition}

The results contain seven species, whose presence indicate the extension of their geographical repartition. In these species, Upupa epops is non Passerine bird and the others are breeding birds.

\subsection{Migratory Birds}

Some migrating species were recorded among the birds living in or transiting by Kinshasa. In this case, there are numerous birds belonging to different orders, like Pelecaniformes as Herons, Strigiformes as Tyto alba, Falconiformes as Falco tinnunculus and Passeriformes as Sylvia borin and Motacilla flava.

\subsection{Conservation}

Only two species-grey parrot (Psittachus erythacus) and Western cattle erget (Bubulcus ibis) are included in the Congolese law on hunting (law No. 
Table 1 Updated names of the avian.

\begin{tabular}{|c|c|c|c|}
\hline \multirow{2}{*}{ No. } & \multirow{2}{*}{ Family } & \multicolumn{2}{|c|}{ Species } \\
\hline & & {$[7]^{*}$} & {$[30-32]^{* *}$} \\
\hline 1 & Phalacrocoracidae & 1 Phalacrocorax africanus africanus & Microcarbo africanus \\
\hline 2 & Ardeidae & 2 Pyrrhorodia purpurea purpurea & Ardea purpurea \\
\hline 3 & Scopidae & 3 Scopus ombretta Bannermanni & Scopus umbretta \\
\hline 4 & Ciconiidae & 4 Ibis ibis & Mycteria ibis \\
\hline 5 & Rallidae & 5 Limnocorax flavirostris & Amaurornis flavirostra \\
\hline 6 & Scolopacidae & 6 Actitis hypoleuca & Actitis hypoleucos \\
\hline \multirow{2}{*}{7} & \multirow{2}{*}{ Glareolidae } & 7 Galachrysia nuchalis Emini & Glareola nuchalis \\
\hline & & 8 Galachrysia cinerea & Glareola cinerea \\
\hline 8 & Jacanidae & 9 Actophilus africanus & Actophilornis africanus \\
\hline 9 & Columbidae & 10 Vinago clava calva & Treron calvus \\
\hline 10 & Cuculidae & 11 Chrysococcyx cupeus intermedius & Chrysococcyx caprius \\
\hline 11 & Coraciidae & 12 Eurystomus afer afer & Eurystomus glaucurus \\
\hline \multirow{2}{*}{12} & \multirow{2}{*}{ Meropidae } & 13 Melittophagus pusillus meridionalis & Merops pusillus \\
\hline & & 14 Meropogon breweri & Merops breweri \\
\hline \multirow{2}{*}{13} & \multirow{2}{*}{ Bucerotidae } & 15 Lophoceros fasciatus. & Tockus fasciatus \\
\hline & & 16 Bycanistes sharpei & Bycanistes fistulator \\
\hline 14 & Apodidae & 17 Tachornis parvus brachypterus & Cypsiurus parvus \\
\hline 15 & Coliidae & 18 Colius nigricollis nigricollis & Colius striatus \\
\hline \multirow{2}{*}{16} & \multirow{2}{*}{ Picidae } & 19 Campethera permista & Campethera cailliautii \\
\hline & & 20 Dendropicos lafresnayi lafresnayi & Dendropicos fuscescens \\
\hline 17 & Sylviidae & 21 Sylvia borin borin & Sylvia borin \\
\hline \multirow{4}{*}{18} & \multirow{4}{*}{ Cisticolidae } & 22 Burnesia leucopogon leucopogon & Schistolais leucopogon \\
\hline & & 23 Cisticola nalensis & Cisticola natalensis \\
\hline & & 24 Cisticola rufocapileata & Cisticola fulvicapilla \\
\hline & & 25 Eremomela scotops mentalis & Eremomela scotops \\
\hline \multirow{2}{*}{19} & \multirow{2}{*}{ Muscicapidae } & 26 Bradornis murinus & Melaenornis pallidus \\
\hline & & 27 Cichladusa ruficauda & Cichladusa ruficauda \\
\hline 20 & Turdidae & 28 Turdus libonyanus saturatus & Turdus libonyana \\
\hline 21 & Hirundinidae & 29 Hirundo puella unitatis & Cercropis abyssinica \\
\hline 22 & Malaconotidae & 30 Tschagra senegala rufofusca & Tchagra senegalus \\
\hline 23 & Laniidae & 31 Fiscus collaris congicus & Lanius collaris \\
\hline 24 & Paridae & 32 Parus niger leucomelas & Melaniparus leucomelas \\
\hline 25 & Nectariniidae & 33 Anthreptes collaris hypodilus & Hedydipna collaris \\
\hline \multirow{4}{*}{26} & \multirow{4}{*}{ Ploceidae } & 34 Hyphantornis collaris & Ploceus cucullatus \\
\hline & & 35 Melanopteryx nigerrimus & Ploceus nigerrimus \\
\hline & & 36 Pyromelana hordacea & Euplectes hordeaceus \\
\hline & & 37 Sitagra monacha & Ploceus pelzelni \\
\hline \multirow{2}{*}{27} & \multirow{2}{*}{ Viduidae } & 38 Coliuspasser macroura macroura & Vidua macroura \\
\hline & & 39 Vidua serena & Emberiza sp. \\
\hline 28 & Passeridae & 40 Passer griseus griseus & Passer griseus \\
\hline
\end{tabular}

[7]*Means names of families and species of birds were assigned by authors in Ref. [7]; [30-32]**means names of families and species of birds were assigned by authors in Refs. [30-32]. 
Table 2 List of current species of the birds of Kinshasa.

\begin{tabular}{|c|c|c|c|}
\hline No. & Oder & Family & Species \\
\hline \multirow[t]{3}{*}{1} & Galliformes & 1 Pasianidae & 1 Pternistis afer ( Statius Müller, 1776) \\
\hline & & 2 Threskiornithidae & 2 Plegadis falcinellus ${ }^{*}$ (Linnaeus, 1766) \\
\hline & & & 3 Ardea cinerea* (Linnaeus, 1758) \\
\hline \multirow[t]{3}{*}{2} & Pelecaniformes & 3 Ardeidae & 4 Ardea melanocephala (Vigors \& Children, 1826) \\
\hline & & & 5 Ardea purpurea (Linnaeus, 1766) \\
\hline & & & 6 Bubulcus ibis ${ }^{* * *}$ (Linnaeus, 1758) \\
\hline 3 & Gruiformes & 4 Rallidae & 7 Porphyrio alleni (Thomson, 1842) \\
\hline \multirow[t]{2}{*}{4} & Charadriiformes & 5 Chara driigae & 8 Charadrius hiaticula (Lowe, 1915) \\
\hline & & & 9 Gypohierax angolensis (Gmelin, 1788) \\
\hline \multirow[t]{3}{*}{5} & Accipitriformes & 6 Accipitridae & 10 Milvus aegyptius (Gemlin, 1788) \\
\hline & & & 11 Milvus migrans (Boddaert, 1783) \\
\hline & & & 12 Streptopelia semitorquata (Ruppell, 1837) \\
\hline \multirow{4}{*}{6} & Columbiforme & 7 Columbido & 13 Turtur afer (Linné, 1766) \\
\hline & Columbiformes & / Columbidae & 14 Treron calvus (Temminick \& Knip, 1809) \\
\hline & & & 15 Turtur tympanistria (Temminick, 1810) \\
\hline & & & 16 Centropus senegalensis (Linnaeus, 1766) \\
\hline \multirow[t]{2}{*}{7} & Cuculiformes & 8 Cuculidae & 17 Ceuthmochares aerus (Vieillot, 1817) \\
\hline & & & 18 Chrysococcys caprius (Boddaert, 1783) \\
\hline \multirow{2}{*}{8} & & 9 Tytonidae & 19 Tyto alba (Fraser, 1842) \\
\hline & Strigiformes & 10 Strigidae & 20 Bubo poensis (Fraser, 1853) \\
\hline 9 & Apodiformes & 11 Apodidae & 21 Apus batesi (Sharpe, 1904) \\
\hline \multirow[t]{4}{*}{10} & Coliiformes & 12 Coliidae & 22 Colius striatus (Gmelin, 1789) \\
\hline & & & 23 Ceryle rudis (Linnaeus, 1758) \\
\hline & & & 24 Corythornis cristatus (Pallas, 1764) \\
\hline & & & 25 Halcyon albiventris (Scopoli, 1868) \\
\hline \multirow{5}{*}{11} & Cornoiformes & 13 Alcedinidae & 26 Halcyon leucocephala (Müller, 1776) \\
\hline & Coraci1formes & & 27 Halcyon senegalensis (Linnaeus, 1766) \\
\hline & & & 28 Ispidina lecontei (Cassin, 1857) \\
\hline & & & 29 Ispidina picta (Boddaert, 1783) \\
\hline & & 14 Meropidae & 30 Merops pusillus (Müller, 1776) \\
\hline \multirow[t]{6}{*}{12} & Bucerotiformes & 15 Upupidae & 31 Upupa africana (Bechstein, 1811) \\
\hline & & & 32 Upupa epops $^{* *}$ (Linnaeus, 1758) \\
\hline & & & 33 Campethera abingoni (Smith, 1837) \\
\hline & & & 34 Campethera cailliautii (Malherbe, 1900) \\
\hline & & 16 Di id & 35 Campethera nivosa (Swainson, 1837) \\
\hline & & 16 Picidae & 36 Dendropicos fuscescens (Vieillot, 1849) \\
\hline \multirow[t]{5}{*}{13} & Piciformes & & 37 Dendropicos gabonensis (Verreaux, 1851) \\
\hline & & & 38 Jynx ruficollis (Wagler, 1830) \\
\hline & & & 39 Lybius minor (Cuvier, 1817) \\
\hline & & 17 Lybiidae & 40 Pogoniulius bilineatus (Sundervll, 1897) \\
\hline & & & 41 Pogoniulius scolopaceus (Bonaparte, 1850) \\
\hline \multirow[t]{2}{*}{14} & Falconiformes & 18 Falconidae & 42 Falco tinnunculus $^{*}$ (Linnaeus, 1758) \\
\hline & & & 43 Agapornis pullarius (Linnaeus, 1758) \\
\hline \multirow[t]{2}{*}{15} & Psittaciformes & 19 Psittacidae & 44 Psittacus erithacus ${ }^{* * *}$ (Linnaeus, 1758) \\
\hline & & & 45 Poicephalus gulielmi (Jardine, 1849) \\
\hline
\end{tabular}


(Table 2 continued)

\begin{tabular}{|c|c|c|c|}
\hline No. & Oder & Family & Species \\
\hline \multirow{44}{*}{16} & \multirow{44}{*}{ Passeriformes } & \multirow{3}{*}{20 Platysteridae } & 46 Batis minor (Neuman, 1907) \\
\hline & & & 47 Batis molitor (Hahn \& Kuster, 1850) \\
\hline & & & 48 Platysteira cyanea (Müller, 1776) \\
\hline & & \multirow{2}{*}{21 Malaconotidae } & 49 Laniarius bicolor (Verreaux, 1857) \\
\hline & & & 50 Laniarius leucorhynchus (Hartlaub, 1848) \\
\hline & & \multirow[t]{2}{*}{22 Laniidae } & 51 Lanius collaris (Linnaeus, 1758) \\
\hline & & & 52 Terpsiphone batesi (Chapin, 1921) \\
\hline & & \multirow[t]{2}{*}{23 Monarchidae } & 53 Terpsiphone rufocinerea (Cabanis, 1875) \\
\hline & & & 54 Terpsiphone viridis (Müller, 1776) \\
\hline & & 24 Corvidae & 55 Corvus albus (Müller, 1776) \\
\hline & & \multirow[t]{5}{*}{25 Paridae } & 56 Melaniparus rufiventris (Bocage, 1885) \\
\hline & & & 57 Atimalistillas flavicollis (Swainson, 1837) \\
\hline & & & 58 Chlorocichla falkensteini (Reichenow, 1874) \\
\hline & & & 59 Chlorocichla simplex (Hartlaub, 1855) \\
\hline & & & 60 Criniger barbatus $^{* *}$ (Temminck, 1821$)$ \\
\hline & & \multirow{6}{*}{26 Pycnonotidae } & 61 Eurrillas virens (Cassin, 1857) \\
\hline & & & 62 Neolestes torquatus (Cabanis, 1875) \\
\hline & & & 63 Phyllastrephus icterinus (Bonaparte, 1850) \\
\hline & & & 64 Phyllastrephus scandens (Swainson, 1837) \\
\hline & & & 65 Pycnonotus barbatus (Desfontaines, 1789) \\
\hline & & & 66 Pycnonotus tricolor (Desfontaines, 1789) \\
\hline & & & 67 Cecropis senegalensis (Linnaeus, 1766) \\
\hline & & \multirow[t]{2}{*}{27 Hirundinidae } & 68 Hirundo rustica (Linnaeus, 1758) \\
\hline & & & 69 Psalidopcorne pristoptera (Ruppell, 1836) \\
\hline & & 28 Macrosphenidae & 70 Melocichla mentalis $^{* *}($ Fraser, 1843) \\
\hline & & \multirow[t]{4}{*}{29 Acrocephalidae } & 71 Acrocephalus rufescens (Sharpe \& Bouvier, 1876) \\
\hline & & & 72 Calamonastes undosus (Reichenow, 1882) \\
\hline & & & 73 Camaroptera brevicaudata (Cretzschmar, 1831) \\
\hline & & & 74 Cisticola brachypterus (Sharpe, 1890) \\
\hline & & \multirow[t]{4}{*}{30 Cisticolidae } & 75 Cisticola erythrops (Hartlaub, 1857) \\
\hline & & & 76 Eremomela icteropygialis ** (Lafresnaye, 1839) \\
\hline & & & 77 Prinia bairdii (Cassin, 1855) \\
\hline & & & 78 Prinia subflava (Gmelin, 1789) \\
\hline & & 31 Sylviidae & 79 Sylvia borin* (Boddaert, 1783) \\
\hline & & \multirow[t]{5}{*}{32 Turdidae } & 80 Turdus pelios (Bonaparte, 1850) \\
\hline & & & 81 Cercotrichas barbata** (Hartlaub \& Finsch, 1870) \\
\hline & & & 82 Cercotrichas hartlaubi (Reichenow, 1891) \\
\hline & & & 83 Cichladusa ruficauda (Hartlaub, 1857) \\
\hline & & & 84 Cossypha niveicapilla (Lafresnaye, 1838) \\
\hline & & \multirow[t]{5}{*}{33 Muscicapidae } & 85 Melaenornis pallidus (Von Müller, 1851) \\
\hline & & & 86 Muscicapa comitata (Cassin, 1857) \\
\hline & & & 87 Myrmecocichla nigra (Vieillot, 1818) \\
\hline & & & 88 Oenanthe familiaris (Wilkes, 1817) \\
\hline & & & 89 Saxicola torquatus (Linnaeus, 1758) \\
\hline
\end{tabular}


(Table 2 continued)

\begin{tabular}{|c|c|c|c|}
\hline No. & Oder & Family & Species \\
\hline & & \multirow{9}{*}{34 Nectariniidae } & 90 Anthreptes longuemarei (Lesson, 1831) \\
\hline & & & 91 Chalcomitra fuliginosa (Bechstein, 1811) \\
\hline & & & 92 Chalcomitra senegalensis (Linnaeus, 1766) \\
\hline & & & 93 Cinnyris chloropygius (Jardine, 1842) \\
\hline & & & 94 Cinnyris cupreus (Shaw, 1811) \\
\hline & & & 95 Cinnyris venustus (Shaw, 1799) \\
\hline & & & 96 Cyanomitra cyanolaema (Jardine \& Fraser, 1851) \\
\hline & & & 97 Cyanomitra olivacea (Smith, 1840) \\
\hline & & & 98 Cyanomitra verticalis (Latham, 1790) \\
\hline & & \multirow[t]{2}{*}{35 Passeridae } & 99 Passer griseus (Vieillot, 1817) \\
\hline & & & 100 Brachycope anomala (Reichenow, 1887) \\
\hline & & \multirow{11}{*}{36 Ploceidae } & 101 Euplectes afer (Gmelin, 1789) \\
\hline & & & 102 Euplectes ardens (Boddaert, 1783) \\
\hline & & & 103 Euplectes gierowii (Cabanis, 1880) \\
\hline & & & 104 Euplectes hordeaceus (Linnaeus, 1758) \\
\hline & & & 105 Euplectes macrourus (Gmelin, 1789) \\
\hline & & & 106 Ploceus cucullatus (Müller, 1776) \\
\hline & & & 107 Ploceus intermedius (Ruppel, 1845) \\
\hline & & & 108 Ploceus nigerrimus (Reichenow, 1904) \\
\hline & & & 109 Ploceus nigricollis (Vieillot, 1805) \\
\hline & & & 110 Ploceus nigrimentus (Reichenow, 1904) \\
\hline & & & 111 Ploceus pelzelni (Hartlaub, 1887) \\
\hline & & \multirow{14}{*}{37 Estrildidae } & 112 Ploceus velatus ${ }^{* *}$ (Vieillot, 1819) \\
\hline & & & 113 Clytospiza monteiri (Hartlaub, 1860) \\
\hline & & & 114 Estrilda astrild (Linnaeus, 1758) \\
\hline & & & 115 Estrilda melpoda (Vieillot, 1817) \\
\hline & & & 116 Lagonosticta rubricata (Lichtenstein, 1868) \\
\hline & & & 117 Pyrenestes ostrinus (Vieillot, 1805) \\
\hline & & & 118 Pytilia afra (Gmelin, 1789) \\
\hline & & & 119 Lonchura bicolor (Fraser, 1843) \\
\hline & & & 120 Lonchura cucullata (Swainson, 1837) \\
\hline & & & 121 Lonchura fringilloides (Lafresnaye, 1835) \\
\hline & & & 122 Spermophaga haematina (Vieillot, 1807) \\
\hline & & & 123 Spermophaga ruficapilla (Shelley, 1888) \\
\hline & & & 124 Uraeginthus angolensis (Linnaeus, 1758) \\
\hline & & & 125 Uraeginthus bengalus $^{* *}$ (Linnaeus, 1766) \\
\hline & & \multirow{2}{*}{38 Viduidae } & 126 Anomalospiza imberbis (Cabanis, 1868) \\
\hline & & & 127 Vidua macroura (Pallas, 1764) \\
\hline & & \multirow{2}{*}{39 Motacillidae } & 128 Motacilla aguimp (Dumont, 1850) \\
\hline & & & 129 Motacilla flava* (Linnaeus, 1758) \\
\hline & & \multirow{2}{*}{40 Fringilidae } & 130 Crithagra capistratus (Finsch, 1870) \\
\hline & & & 131 Crithagra mozambica (Statius Müller, 1776) \\
\hline
\end{tabular}

*Means migrating; **means widening of the geographical distribution; $* * *$ means protected species.

82-002 of May 28, 1982). Based on this law and with respect to Washington Convention of March 03, 1973, $P$. erythacus is on list one and B. ibis is on list two. All others birds are subject to exploitation.

\subsection{Evolution}

In term of evolution, it has to be pointed out that changes occurred over time in the composition and 
diversity of birds in Kinshasa. Table 3 indicates that main changes happened in two patterns. Out of 170 birds, $41(24.12 \%)$ species are omnipresent, 39 (22.94\%) species were not observed in these last years and 90 (52.94\%) are new incoming birds. As we can see, half of actual birds, Passerines as well as non-Passerine of Kinshasa are mainly constituted with new species, all of which were subjected to changes.

\section{Discussion}

\subsection{Composition and Diversity}

The number of bird's species currently recorded in Kinshasa is higher than 111 species [8] previously reported. The number of birds in DRC vary with authors: 1064 [33], 1086 [22], 1099 [34], 1118 [35], 1149 [36], 1176 [37], 1180 [31], 1181 [38], 1185 [39]. According to these numbers, data from the research indicate that about $11 \%$ of the birds of DRC live in
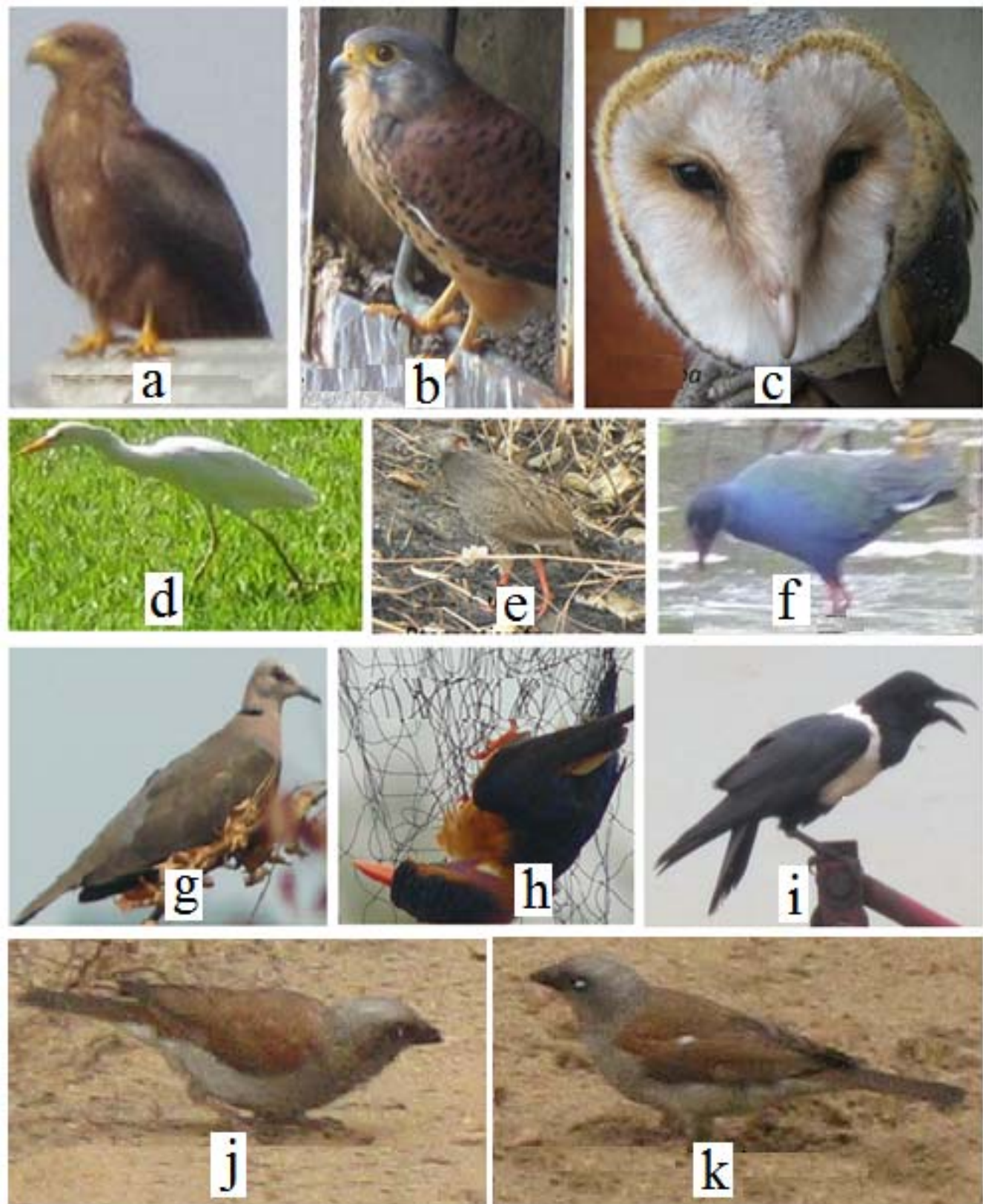

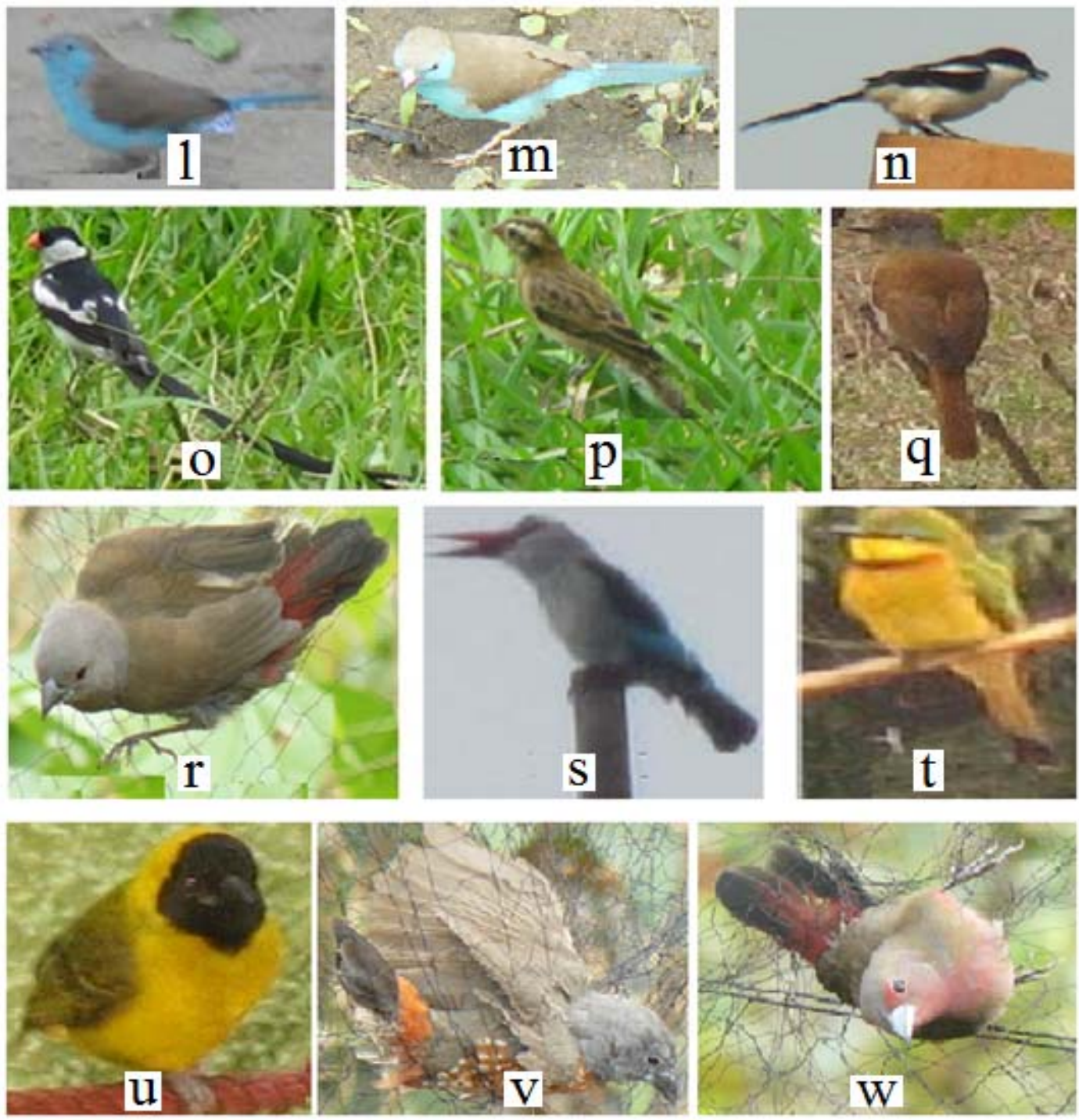

Fig. 1 Few birds of Kinshasa, Democratic Republic of Congo.

a-Milvus aegyptius; b—Falco tinunculus; c—Tyto alba (juvenil); d-Bubulcus ibis; e—Pternistis afer; f—Porphyrio alleni; g-Streptopelia semitorquata; $\mathrm{h}$-Ispidina picta; $\mathrm{i}$-Corvus albus; $\mathrm{j}$-Passer griseus; $\mathrm{k}$-Passer griseus; 1 -Uraeginthus angolensis; $\mathrm{m}$-Uraeginthus angolensis; n-Laniarius bicolor; o-Vidua macroura (male); p-Vidua macroura (female); q-Cichladusa ruficauda; $\mathrm{r}$-Estrilda perreini; $\mathrm{s}-$ Halcyon senegalensis; $\mathrm{t}$-Merops variegatus; $\mathrm{u}-$ Plocceus pelzelni; $\mathrm{v}-$ Clytospiza monteiri; $\mathrm{w}$-Lagonostica rubricate.

Kinshasa shelters. The authors believe that this report could be improved in the future. Among the listed species, Passerines are the most representative in diversity of species and they include 86 species which account for $66 \%$ of birds. Comparing present data with others [22], the Passerines of Kinshasa represent $14 \%$ of species of those of the whole country. In this order, only six among the 21 families recorded are well represented. In a decreasing order, they are Estrildidae, Ploceidae, Pychnonotidae, Muscicapidae,
Nectarinidae and Cisticolidae. These six families together represent 61 species, i.e., $70.9 \%$ of species of the Passerines of Kinshasa.

According to the current data, none of the species known as endemic in DRC has been observed so far in Kinshasa.

\subsection{Geographical Distribution}

There is a difference in opinion of several authors as to geographical distribution of some species of 
Table 3 Checklist of birds of Kinshasa.

\begin{tabular}{|c|c|c|c|c|}
\hline No. & Species & {$[7]$} & [8] & This study \\
\hline 1 & Ardea melanocephala & $\mathrm{x}$ & $\mathrm{x}$ & $\mathrm{x}$ \\
\hline 2 & Atimastillas flavicollis & $\mathrm{x}$ & $\mathrm{x}$ & $\mathrm{x}$ \\
\hline 3 & Bubulcus ibis & $\mathrm{x}$ & $\mathrm{x}$ & $\mathrm{x}$ \\
\hline 4 & Campethera cailliautii & $\mathrm{x}$ & $\mathrm{x}$ & $\mathrm{x}$ \\
\hline 5 & Ceuthmochares aereus & $\mathrm{x}$ & $\mathrm{x}$ & $\mathrm{x}$ \\
\hline 6 & Chrysococcyх cupreus & $\mathrm{x}$ & $\mathrm{x}$ & $\mathrm{x}$ \\
\hline 7 & Cichladusa ruficauda & $\mathrm{x}$ & $\mathrm{x}$ & $\mathrm{x}$ \\
\hline 8 & Cinnyris cupreus & $\mathrm{x}$ & $\mathrm{x}$ & $\mathrm{x}$ \\
\hline 9 & Colius striatus & $\mathrm{x}$ & $\mathrm{x}$ & $\mathrm{x}$ \\
\hline 10 & Cyanomitra verticalis & $\mathrm{x}$ & $\mathrm{x}$ & $\mathrm{x}$ \\
\hline 11 & Dendropicos fuscescens & $\mathrm{x}$ & $\mathrm{x}$ & $\mathrm{x}$ \\
\hline 12 & Estrilda melpoda & $\mathrm{x}$ & $\mathrm{x}$ & $\mathrm{x}$ \\
\hline 13 & Euplectes hordeaceus & $\mathrm{x}$ & $\mathrm{x}$ & $\mathrm{x}$ \\
\hline 14 & Eurillas virens & $\mathrm{x}$ & $\mathrm{x}$ & $\mathrm{x}$ \\
\hline 15 & Halcyon senegalensis & $\mathrm{x}$ & $\mathrm{x}$ & $\mathrm{x}$ \\
\hline 16 & Lanius collaris & $\mathrm{x}$ & $\mathrm{x}$ & $\mathrm{x}$ \\
\hline 17 & Melaenornis pallidus & $\mathrm{x}$ & $\mathrm{x}$ & $\mathrm{x}$ \\
\hline 18 & Merops pusillus & $\mathrm{x}$ & $\mathrm{x}$ & $\mathrm{x}$ \\
\hline 19 & Milvus aegyptius & $\mathrm{x}$ & $\mathrm{x}$ & $\mathrm{x}$ \\
\hline 20 & Neolestes torquatus & $\mathrm{x}$ & $\mathrm{x}$ & $\mathrm{x}$ \\
\hline 21 & Passer griseus & $\mathrm{x}$ & $\mathrm{x}$ & $\mathrm{x}$ \\
\hline 22 & Platysteira cyanea & $\mathrm{x}$ & $\mathrm{x}$ & $\mathrm{x}$ \\
\hline 23 & Ploceus cucullatus & $\mathrm{x}$ & $\mathrm{x}$ & $\mathrm{x}$ \\
\hline 24 & Ploceus nigerrimus & $\mathrm{x}$ & $\mathrm{x}$ & $\mathrm{x}$ \\
\hline 25 & Ploceus pelzelni & $\mathrm{x}$ & $\mathrm{x}$ & $\mathrm{x}$ \\
\hline 26 & Psittacus erithacus & $\mathrm{x}$ & $\mathrm{x}$ & $\mathrm{x}$ \\
\hline 27 & Pycnonotus tricolor & $\mathrm{x}$ & $\mathrm{x}$ & $\mathrm{x}$ \\
\hline 28 & Pyrenestes ostrinus & $\mathrm{x}$ & $\mathrm{x}$ & $\mathrm{x}$ \\
\hline 29 & Spermestes cucullatus & $\mathrm{x}$ & $\mathrm{x}$ & $\mathrm{x}$ \\
\hline 30 & Streptopelia semitorquata & $\mathrm{x}$ & $\mathrm{x}$ & $\mathrm{x}$ \\
\hline 31 & Sylvia borin & $\mathrm{x}$ & $\mathrm{x}$ & $\mathrm{x}$ \\
\hline 32 & Terpisphone rufocinerea & $\mathrm{x}$ & $\mathrm{x}$ & $\mathrm{x}$ \\
\hline 33 & Turtur afer & $\mathrm{x}$ & $\mathrm{x}$ & $\mathrm{x}$ \\
\hline 34 & Ardea purpurea & $\mathrm{x}$ & & $\mathrm{x}$ \\
\hline 35 & Centropus senegalensis & $\mathrm{x}$ & & $\mathrm{x}$ \\
\hline 36 & Ceryle rudis & $\mathrm{x}$ & & $\mathrm{x}$ \\
\hline 37 & Gypohierax angolensis & $\mathrm{x}$ & & $\mathrm{x}$ \\
\hline 38 & Motacilla aguimp & $\mathrm{x}$ & & $\mathrm{x}$ \\
\hline 39 & Myrmecocichla nigra & $\mathrm{x}$ & & $\mathrm{x}$ \\
\hline 40 & Treron calvus & $\mathrm{x}$ & & $\mathrm{x}$ \\
\hline 41 & Vidua macroura & $\mathrm{x}$ & & $\mathrm{x}$ \\
\hline 42 & Actitis hypoleucos & $\mathrm{x}$ & & \\
\hline 43 & Actophilornis africanus & $\mathrm{x}$ & & \\
\hline 44 & Amaurornis flavirostra & $\mathrm{x}$ & & \\
\hline 45 & Anhinga rufa & $\mathrm{x}$ & & \\
\hline 46 & Anthreptes collaris & $\mathrm{x}$ & & \\
\hline 47 & Baeopogon indicator & $\mathrm{x}$ & & \\
\hline 48 & Butorides atricapillus & $\mathrm{x}$ & & \\
\hline
\end{tabular}


(Table 3 continued)

\begin{tabular}{|c|c|c|c|c|}
\hline No. & Species & [7] & {$[8]$} & This study \\
\hline 49 & Bycanistes fistulator & $\mathrm{x}$ & & \\
\hline 50 & Cercropis abyssinica & $\mathrm{x}$ & & \\
\hline 51 & Cisticola fulvicapilla & $\mathrm{x}$ & & \\
\hline 52 & Cisticola lateralis & $\mathrm{x}$ & & \\
\hline 53 & Cisticola natalensis & $\mathrm{x}$ & & \\
\hline 54 & Cypsiurus parvus & $\mathrm{x}$ & & \\
\hline 55 & Dendrocygna viduata & $\mathrm{x}$ & & \\
\hline 56 & Dicrurus modestus & $\mathrm{x}$ & & \\
\hline 57 & Dryoscopus gambensis & $\mathrm{x}$ & & \\
\hline 58 & Emberiza sp. & $\mathrm{x}$ & & \\
\hline 59 & Eremomela scotops & $\mathrm{x}$ & & \\
\hline 60 & Eurystomus glaucurus & $\mathrm{x}$ & & \\
\hline 61 & Glareola cinerea & $\mathrm{x}$ & & \\
\hline 62 & Glareola nuchalis & $\mathrm{x}$ & & \\
\hline 63 & Kaupifalco monogramicus & $\mathrm{x}$ & & \\
\hline 64 & Larus fuscus & $\mathrm{x}$ & & \\
\hline 65 & Macronyx croceus & $\mathrm{x}$ & & \\
\hline 66 & Megaceryle maxima & $\mathrm{x}$ & & \\
\hline 67 & Melaniparus leucomelas & $\mathrm{x}$ & & \\
\hline 68 & Merops breweri & $\mathrm{x}$ & & \\
\hline 69 & Merops malimbicus & $\mathrm{x}$ & & \\
\hline 70 & Microcarbo africanus & $\mathrm{x}$ & & \\
\hline 71 & Mycteria ibis & $\mathrm{x}$ & & \\
\hline 72 & Nicator chloris & $\mathrm{x}$ & & \\
\hline 73 & Pelecanus onocrotalus & $\mathrm{x}$ & & \\
\hline 74 & Pogoniulus subsulphureus & $\mathrm{x}$ & & \\
\hline 75 & Rynchops flavirostris & $\mathrm{x}$ & & \\
\hline 76 & Schistolais leucopogon & $\mathrm{x}$ & & \\
\hline 77 & Scopus umbreta & $\mathrm{x}$ & & \\
\hline 78 & Tchagra senegalus & $\mathrm{x}$ & & \\
\hline 79 & Tockus fasciatus & $\mathrm{x}$ & & \\
\hline 80 & Turdus libonyanus & $\mathrm{x}$ & & \\
\hline 81 & Acrocephalus rufescens & & $\mathrm{x}$ & $\mathrm{x}$ \\
\hline 82 & Agapornis pullarius & & $\mathrm{x}$ & $\mathrm{x}$ \\
\hline 83 & Alcedo cristata & & $\mathrm{x}$ & $\mathrm{x}$ \\
\hline 84 & Anomalospiza imberbis & & $\mathrm{x}$ & $\mathrm{x}$ \\
\hline 85 & Anthreptes longuemarei & & $\mathrm{x}$ & $\mathrm{x}$ \\
\hline 86 & Apus batesi & & $\mathrm{x}$ & $\mathrm{x}$ \\
\hline 87 & Batis minor & & $\mathrm{x}$ & $\mathrm{x}$ \\
\hline 88 & Batis molitor & & $\mathrm{x}$ & $\mathrm{x}$ \\
\hline 89 & Brachycope anomala & & $\mathrm{x}$ & $\mathrm{x}$ \\
\hline 90 & Camoroptera brevicaudata & & $\mathrm{x}$ & $\mathrm{x}$ \\
\hline 91 & Campethera abingoni & & $\mathrm{x}$ & $\mathrm{x}$ \\
\hline 92 & Campethera nivosa & & $\mathrm{x}$ & $\mathrm{x}$ \\
\hline 93 & Cercomela familiaris & & $\mathrm{x}$ & $\mathrm{x}$ \\
\hline 94 & Chalcomitra fuliginosa & & $\mathrm{x}$ & $\mathrm{x}$ \\
\hline 95 & Chalcomitra senegalensis & & $\mathrm{x}$ & $\mathrm{x}$ \\
\hline 96 & Charadrius hiaticula & & $\mathrm{x}$ & $\mathrm{x}$ \\
\hline
\end{tabular}




\begin{tabular}{|c|c|c|c|c|}
\hline No. & Species & {$[7]$} & [8] & This study \\
\hline 97 & Chlorocichla falkensteini & & $\mathrm{x}$ & $\mathrm{x}$ \\
\hline 98 & Chlorocichla simplex & & $\mathrm{x}$ & $\mathrm{x}$ \\
\hline 99 & Cinnyris chloropygius & & $\mathrm{x}$ & $\mathrm{x}$ \\
\hline 100 & Cinnyris venustus & & $\mathrm{x}$ & $\mathrm{x}$ \\
\hline 101 & Cisticola brachypterus & & $\mathrm{x}$ & $\mathrm{x}$ \\
\hline 102 & Cisticola erythrops & & $\mathrm{x}$ & $\mathrm{x}$ \\
\hline 103 & Clytospiza monteiri & & $\mathrm{x}$ & $\mathrm{x}$ \\
\hline 104 & Corvus albus & & $\mathrm{x}$ & $\mathrm{x}$ \\
\hline 105 & Cossypha niveicapilla & & $\mathrm{x}$ & $\mathrm{x}$ \\
\hline 106 & Criniger barbatus & & $\mathrm{x}$ & $\mathrm{x}$ \\
\hline 107 & Crithagra capistrata & & $\mathrm{x}$ & $\mathrm{x}$ \\
\hline 108 & Crithagra mozambica & & $\mathrm{x}$ & $\mathrm{x}$ \\
\hline 109 & Cyanomitra cyanolaema & & $\mathrm{x}$ & $\mathrm{x}$ \\
\hline 110 & Cyanomitra olivacea & & $\mathrm{x}$ & $\mathrm{x}$ \\
\hline 111 & Dendropicos gabonensis & & $\mathrm{x}$ & $\mathrm{x}$ \\
\hline 112 & Eremomela icteropygialis & & $\mathrm{x}$ & $\mathrm{x}$ \\
\hline 113 & Erythropygia barbata & & $\mathrm{x}$ & $\mathrm{x}$ \\
\hline 114 & Erythropygia hartlaubi & & $\mathrm{x}$ & $\mathrm{x}$ \\
\hline 115 & Estrilda astrild & & $\mathrm{x}$ & $\mathrm{x}$ \\
\hline 116 & Euplectes afer & & $\mathrm{x}$ & $\mathrm{x}$ \\
\hline 117 & Euplectes ardens & & $\mathrm{x}$ & $\mathrm{x}$ \\
\hline 118 & Euplectes gierowii & & $\mathrm{x}$ & $\mathrm{x}$ \\
\hline 119 & Euplectes macrourus & & $\mathrm{x}$ & $\mathrm{x}$ \\
\hline 120 & Halcyon albiventris & & $\mathrm{x}$ & $\mathrm{x}$ \\
\hline 121 & Halcyon leucocephala & & $\mathrm{x}$ & $\mathrm{x}$ \\
\hline 122 & Hirundo rustica & & $\mathrm{x}$ & $\mathrm{x}$ \\
\hline 123 & Ispidina lecontei & & $\mathrm{x}$ & $\mathrm{x}$ \\
\hline 124 & Ispidina picta & & $\mathrm{x}$ & $\mathrm{x}$ \\
\hline 125 & Jynx ruficollis & & $\mathrm{x}$ & $\mathrm{x}$ \\
\hline 126 & Lagonosticta rubricate & & $\mathrm{x}$ & $\mathrm{x}$ \\
\hline 127 & Laniarius bicolor & & $\mathrm{x}$ & $\mathrm{x}$ \\
\hline 128 & Laniarius leucorhynchus & & $\mathrm{x}$ & $\mathrm{x}$ \\
\hline 129 & Lybius minor & & $\mathrm{x}$ & $\mathrm{x}$ \\
\hline 130 & Melocichla mentalis & & $\mathrm{x}$ & $\mathrm{x}$ \\
\hline 131 & Milvus migrans & & $\mathrm{x}$ & $\mathrm{x}$ \\
\hline 132 & Motacilla flava & & $\mathrm{x}$ & $\mathrm{x}$ \\
\hline 133 & Muscicapa comitata & & $\mathrm{x}$ & $\mathrm{x}$ \\
\hline 134 & Parus rufiventris & & $\mathrm{x}$ & $\mathrm{x}$ \\
\hline 135 & Phyllastrephus icterinus & & $\mathrm{x}$ & $\mathrm{x}$ \\
\hline 136 & Ploceus intermedius & & $\mathrm{x}$ & $\mathrm{x}$ \\
\hline 137 & Ploceus nigricollis & & $\mathrm{x}$ & $\mathrm{x}$ \\
\hline 138 & Ploceus nigrimentus & & $\mathrm{x}$ & $\mathrm{x}$ \\
\hline 139 & Ploceus velatus & & $\mathrm{x}$ & $\mathrm{x}$ \\
\hline 140 & Pogoniulius bilineatus & & $\mathrm{x}$ & $\mathrm{x}$ \\
\hline 141 & Pogoniulius scolopaceus & & $\mathrm{x}$ & $\mathrm{x}$ \\
\hline 142 & Poicephalus gulielmi & & $\mathrm{x}$ & $\mathrm{x}$ \\
\hline 143 & Porphyrio alleni & & $\mathrm{x}$ & $\mathrm{x}$ \\
\hline 144 & Prinia subflava & & $\mathrm{x}$ & $\mathrm{x}$ \\
\hline
\end{tabular}


(Table 3 continued)

\begin{tabular}{|c|c|c|c|c|}
\hline No. & Species & {$[7]$} & {$[8]$} & This study \\
\hline 145 & Pternistis afer & & $\mathrm{x}$ & $\mathrm{x}$ \\
\hline 146 & Pycnonotus barbatus & & $\mathrm{x}$ & $\mathrm{x}$ \\
\hline 147 & Pytilia afra & & $\mathrm{x}$ & $\mathrm{x}$ \\
\hline 148 & Spermestes bicolor & & $\mathrm{x}$ & $\mathrm{x}$ \\
\hline 149 & Spermestes fringilloides & & $\mathrm{x}$ & $\mathrm{x}$ \\
\hline 150 & Spermophaga haematina & & $\mathrm{x}$ & $\mathrm{x}$ \\
\hline 151 & Spermophaga ruficapilla & & $\mathrm{x}$ & $\mathrm{x}$ \\
\hline 152 & Turdus pelios & & $\mathrm{x}$ & $\mathrm{x}$ \\
\hline 153 & Turtur tympanistria & & $\mathrm{x}$ & $\mathrm{x}$ \\
\hline 154 & Tyto alba & & $\mathrm{x}$ & $\mathrm{x}$ \\
\hline 155 & Upupa africana & & $\mathrm{x}$ & $\mathrm{x}$ \\
\hline 156 & Upupa epops & & $\mathrm{x}$ & $\mathrm{x}$ \\
\hline 157 & Uraeginthus angolensis & & $\mathrm{x}$ & $\mathrm{x}$ \\
\hline 158 & Uraeginthus bengalus & & $\mathrm{x}$ & $\mathrm{x}$ \\
\hline 159 & Ardea cinerea & & & $\mathrm{x}$ \\
\hline 160 & Bubo poensis & & & $\mathrm{x}$ \\
\hline 161 & Calamonastes undosus & & & $\mathrm{x}$ \\
\hline 162 & Cecropis senegalensis & & & $\mathrm{x}$ \\
\hline 163 & Falco tinnunculus & & & $\mathrm{x}$ \\
\hline 164 & Plegadis falcinellus & & & $\mathrm{x}$ \\
\hline 165 & Phyllastrephus scandens & & & $\mathrm{x}$ \\
\hline 166 & Prinia bairdii & & & $\mathrm{x}$ \\
\hline 167 & Psalidopcorne pristoptera & & & $\mathrm{x}$ \\
\hline 168 & Saxicola torquatus & & & $\mathrm{x}$ \\
\hline 169 & Terpsiphone batesi & & & $\mathrm{x}$ \\
\hline \multirow[t]{2}{*}{170} & Terpsiphone viridis & & & $\mathrm{x}$ \\
\hline & Total & 80 & 111 & 131 \\
\hline
\end{tabular}

[7]: species of birds were referred to Ref. [7]; [8]: species of birds were referred to Ref. [8]; this study: species of birds were referred to this study; $x$ : species were noted by the authors.

birds. This is the case of seven species described below. Eremomela icteropygialis (Cisticolidae) for example, is known only in the province of Katanga, while Melocichla mentalis (Macrosphenidae) exists only in the North, East and South of the country [27]. This geographical distribution of the two species could also be extended to the province of Bas-Congo [22]. Generally, Upupa epops (Upupidae) is a species that is largely represented in Africa, except in the forest and desert parts of the continent [22]. However, the picture used for Upupa epops [22] looks like an Upupa africana [27]. In Africa, Upupa epops is a Scandinavian species and its presence could be seen in the Northern part of DRC; while Upupa africana southerner would be inventoried in Kinshasa as well
[27]. The authors observed an individual of Upupa africana at the "Plateau des Residents" (a residential area of the professors of the University of Kinshasa); whereas Upupa epops was observed in Maluku and a specimen of the species was captured in Institut Supérieur Pédagogique (ISP) of Gombe in the middle of the city of Kinshasa. Could this be the same species or two species living together in Kinshasa? This question remains open. The geographical distribution for Uraeginthus begalus (Estrildidae) seems to be limited to the extreme Southern part of the country [27], or "near Kinshasa" [22]; it is one of the most common and regular species in Kinshasa. Ploceus velatus (Ploceidae) is a species confined in the Southern part of Africa; it is reported in the 
Northwestern region of Angola [27]. In DRC, the three subspecies would be limited to the Northeastern and the Southeastern parts of the country [22]. Criniger barbatus (Pycnonotidae) is limited to West Africa $[25,27]$. This species is located only in "large equatorial wet forest at low altitude" [22]. In the area of Kinshasa, the Bulbul was captured in gallery of local forests. The presence of Cercotrichas hartlaubi (Muscicapidae) is dubious in the area of Kinshasa [22, 27].

The presence of all these birds captured or observed in Kinshasa, tells us that these species have widened their geographical distribution.

\subsection{Migratory Birds}

Kinshasa locates on both the East Atlantic byway and the East Africa West Asia byway of migratory birds [40] and receives some Passerine and non-Passerine migrating species at times. Some species of that category must be mentioned. A crew of 10 individuals of glossy ibis (Plegadis falcinellus, Threskiornithidae) was observed during nearly one week in February 2010 in one of the swamps of the Ndjili River slightly at upstream of the bridge which has the same name. Several birds were observed to fly over the airport of Ndolo in West-East direction around 5:00 pm in 2011. Recently in 2013, these birds were seen to whirl above the cemetery of Kinkole at 2:00 pm, probably; they were exploring the surroundings of the Congo River, which is not far from these locations. Those species are found of aquatic habitats [22, 41]. For the garden, warblers (Sylvia borin, Sylviidae) were captured in the grassy zones of Kinshasa region. Herons (Ardeidae) specimens have a large member of migrating species which are regularly observed at river's bank in DRC. [22, 41-43]. The grey heron (Ardea cinerea, Ardeidae) belonged to the species which visited river's bank and marshy zones of the area of Kinshasa. Common kestrel (Falco tinnunculus, Falconidae) is known as Palearctic migratory [27]. Since 1998, some kestrel couples are reproducing each year in building ceilings of the University of Kinshasa. One of the couples chose a broken air-conditioning in the Faculty of Sciences of University of Kinshasa as periodic nesting box, from 2008 to 2012. These examples show that the area of Kinshasa, in spite of its strong human density of 691.55 inhabitants $/ \mathrm{km}^{2}$ in 2005 [10], offers an environment, where some species of birds refuge or use as a temporary location. The regular presence and the reproduction of certain North species in the area of Kinshasa and perhaps also elsewhere in the country are a strong signal which supports this assertion. Do modifications of the reproductive behavior of the migratory birds be related to climate change? Furthermore, do urbanized areas be preferred site for some bird's species?

Although the fact that DRC have ratified both the Ramsar Convention of February 2, 1971 and the Bonn Convention of 1979, none migratory species observed in the town benefits from a particular legal protection.

\subsection{Conservation}

Current text on the conservation of wild fauna in DRC (law No. 82-002 of May 28, 1982 regulating hunting) divides the animal species into three categories as the lists of Washington Convention of March 3, 1973. According to that law, only grey parrot (Psittacus erithacus) appeared on the first list. On the second list, the Western cattle egret (Bubulcus ibis), globally owls and barn owls were found. We thus think that Western barn owl (Tyto alba) is included in this category. No glossy ibis (Plegadis falcinellus) migrating as the African sacred ibis (Threskiornis aethiopicus), was observed in Kinshasa, but selected on this list. All other birds' species belong to the third list (not mentioned) in DRC.

The area of Kinshasa has a hunting reserve at Bombo-Lumene. The reserve was created mainly for hunting mammals especially buffaloes (Syncerus cafer), and not for protecting birds which are suffering from various forms of human exploitation, without 
taking into account their status of conservation.

\subsection{Evolution}

As we see above, the list of known birds in Kinshasa is long and comprise of 170 species (Table 3), which includes Passerines and non-Passerines. That list highlights three principal characteristics. The first category includes species, which occur in the urban environment and therefore are adapted to the human presence for several years. Forty-one species belong to this category (Table 3: from No. 1 to No. 41). Among those species, most are species of open habitats, like savanna and urban places. However, it was observed that the presence of species was also frequently seen in aquatic environments like Ardea melanocephala and in forest habitats like Dendropicos fuscescens. The second category includes species which previously were recorded in Kinshasa, but not observed these last years. They represent half of the species (Table 3: from No. 42 to No. 80). Those species show the same ecological characteristics as those of the first category. They are mentioned that Anhinga rufa, Larus fuscus, Mycteria ibis, Pelecanus onocrotalus and Scopus umbreta for aquatic species and Bycanistes fistulator and Tockus fasciatus for the forest species. On one hand, the presence of the lesser black-backed gull (Larus fuscus) informs us that formerly Malebo Pool has less human presence than it is today, until attracting species to live in coastal zone of the marine environments. On the other hand, the absence of the pied crow (Corvus albus), in the past, omnipresent specie currently in downtown, indicates the degree of degradation of the urban environmental quality of Kinshasa. The third category consists of 90 species (Table 3: from No. 81 to No. 170). In general, the group contains species whose habitats are of similar types of those of the other categories, and in fact Passerines are the most numerous. These facts agree with the idea that birds are good indicators of the state and the health of an ecosystem [44-47]. That situation can be also explained by urban expansion in zones where grassy savannas are the dominant formations of vegetation, as in Maluku, Nsele and the Plateau of Bateke which constitute the peripheral zones of Kinshasa. Let us note that the two municipalities-Maluku and Nsele, are qualified "urbano-rural municipalities" and extend on an area representing $85.8 \%$ of the entire city of Kinshasa [10]. Their vegetation is mainly characterized by a mosaic of savannas, and is favorable habitats for Passerines of open areas. This vegetation is not occurring in the central part of the city. It is the case for the majority of those species. Thus, it is not a new colonization of the urban environment but a consequence related to urban expansion. This enlargement of the town includes new species of birds formerly wild and later forced to cohabit with men.

\section{Conclusions}

Avian fauna of Kinshasa, Democratic Republic of Congo, is rich in number of species and diversity. In actual habitats, this fauna contain species of various ecological statutes. These facts can be due to the lateral extension of town which includes main wild areas.

\section{References}

[1] Chapin, J. P. 1932. "The Birds of the Belgian Congo." American Museum of Natural History 65. Accessed July 16 , 2015. http://www.biodiversitylibrary.org/creator/145633\#titles.

[2] Chapin, J. P. 1939. "The Birds of the Belgian Congo." American Museum of Natural History 75. Accessed July 16 , 2015. http://www.biodiversitylibrary.org/creator/145633\#titles.

[3] Chapin, J. P. 1953. "The Birds of the Belgian Congo." American Museum of Natural History 75 A. Accessed July $16, \quad 2015$. http://www.biodiversitylibrary.org/creator/145633\#titles.

[4] Chapin, J. P. 1954. "The Birds of the Belgian Congo." American Museum of Natural History 75 B. Accessed July $16, \quad 2015$. http://www.biodiversitylibrary.org/creator/145633\#titles.

[5] Schouteden, H. 1957. Fauna of the Belgian Congo and Ruanda-Urundi. Congo: Tervuren Publisher, 328, 459. Accessed July 27, 2015. 
http://www.amazon.com/Faune-Congo-Ruanda-Urundioiseaux-passereaux/dp/B0026NWIEK. (in French)

[6] Verheyen, R. 1955. "The Birds of Belgian Congo.' In Encyclopedia of Belgian Congo. Bruxelles: Tome II Bieleveld, 313-36. (in French)

[7] Schouteden, H. 1924. "Contribution to the Ornithological Fauna of the Belgian Congo: Part III, Data of Kisantu, Leopoldville and Kidada.” Rev. Zool. Afr. 12 (2): 258-77. (in French)

[8] Ifuta, N. B., Kenawabo, T., Mampembe, L., Zambi, T., Palata, K., and Punga, K. 2002. "Avian Fauna of Green Species of Kinshasa and Surroundings." Les Cahiers de l'ISP 11: 19-30. (in French)

[9] Tambwe-Mangala, M. 2010. Democratic Republic of Congo. Bruxelles: Neocity Sprl, 360. (in French)

[10] Lelo-Nzuzi, F. 2008. Kinshasa: Town and Environment. Paris: L'Harmattan, 281. (in French)

[11] Wikipedia. 2014. "Kinshasa." Accessed February 26, 2014. http://fr.wikipedia.org/w/index.php?title=Kinshasa \&oldid=113589001. (in French)

[12] Ministry for Environment and Nature Conservation and Land Affairs. 1999. "Provincial Action Plan of Biodiversity, Kinshasa Province." Accessed July 21, 2015. http://bch-cbd.naturalsciences.be/congodr/cdr-fra/ contribution/strataction/plandaction/provinces.pdf. (in French)

[13] Kottek, M., Grieser, J., Beck, C., Rudolf, B., and Rubel, F. 2006. "World Map of the Koppen-Geiser Climate Classification." Meteorologische Zeitschrift 19 (3): 259-63.

[14] Kalanda, K. 1981. “The Vegetation of N'sele Park.” Jard. Bot. Nat. Belg. 51: 347-57. (in French)

[15] Pauwels, L. 1993. "Guide of Trees and Shrubs of Kinshasa Region and Brazzaville." Nordic Journal of Botany 14 (5): 495. (in French)

[16] Pauwels, L. 2006. "List of Herbaria Specimens near Kinshasa." Catalog of Kinshasa Flora, Herbarium Horti Bot. Nat. Belgii (BR), Progress Database, Spcol, Accessed July 27, 2015. http://www.nzenzeflowerpauwels.be. (in French)

[17] Kikufi, B. 2006. "Floristic and Ecological Study of Massina Swamps (Kinshasa).', M.Sc. thesis, University of Kinshasa. (in French)

[18] Biloso, M. A. 2008. "Valorisation of Non Timbers Products in the Interland of Kinshasa (RDC)." Ph.D. thesis, Faculty of Sciences, Bioingenery Interfacultary School, Free University of Bruxelles. (in French)

[19] Habari, M. J. P. 2009. "Floristic, Phytogeographic and Phytosociology Study of the Vegetation of Kinshasa and Middle Basin of the Rivers of N'djili and N'sele in Democratic Republic of Congo." Ph.D. thesis, Faculty of Sciences, University of Kinshasa. (in French)
[20] Mackworth-Praed, C. W., and Grant, C. H. B. 1960. Birds of Eastern and North Eastern Africa: African Handbook of Birds. Vol. 1. Great Britain: Longmans, 1113.

[21] Mackworth-Praed, C. W., and Grant, C. H. B. 1970. Birds of West Central and Western Africa: African Handbook of Birds. Vol. 3. Great Britain: Longman, 671.

[22] Lippens, L., and Wille, H. 1976. The Birds of Zaïre. Tielt, Belgique: Lannoo International Publisher, 509. (in French)

[23] Girard, O. 1998. Echassiers, Ducks and Sandpipers of West Africa. Le Chateau d'Olone, France: Castel, 136. (in French)

[24] Borrow, N., and Demey, R. 2001. Birds of Western Africa. London: Christopher Helm/A \& C Black Publishers Ltd., 832.

[25] Borrow, N., Demey, R. 2008. Birds of Western Africa. London: Christopher Helm/A \& C Black Publishers Ltd., 511.

[26] Van Perlo, B. 2002. Birds of Western and Central Africa. Princeton, USA: Princeton University Press.

[27] Sinclair, I., and Ryan, P. 2010. Birds of Africa South of the Sahara. Cape Town: Struik Publishers, 767.

[28] Sibley, C. G., and Ahlquist, J. E. 1990. Phylogeny and Classification of Birds: A Study in Molecular Evolution. USA: Yale University Press, 1111.

[29] Lecointre, G., and Le Guyader, H. 2001. Phylogenetic Classification of Living. Paris: Editions Belin. (in French)

[30] Lepage, D. 2007. "Checklist of Birds of the Democratic Republic of Congo." Avibase-Bird Checklists of the World. Accessed November 20, 2014. http://avibase.bsc-eoc.org/checklist.jsp?region $=$ cd\&list $=\mathrm{c}$ lements.

[31] Gill, F., and Donsker, D., ed. 2013. "The Birds of Democratic Republic of Congo." IOC World Bird List Version 4.3. Accessed November 04, 2014. http://www.the-birds.net/aves/republique.democratique.d u.congo.html.

[32] Gill, F., and Donsker, D., ed. 2015. "The Birds of Democratic Republic of Congo.” IOC World Bird List Version 5.1. Accessed April 1, 2015. http://www.worldbirdnames.org/www.worldbirdname.or g.

[33] Environmental Bulletin in Central Africa. 1995. "Special Edito Zaire." Canopee 6: 1-2. Accessed November 14, 2007. http://www.ecofac.org/Canopee/N06/N0600_Edito /ZaireEnvironnement.htm. (in French)

[34] Christy, P., and Van de Weghe, J. P. 1999. The Birds of Central Africa: Faunistical List. Libreville: Association for the Development of Environmental Information (ADIE), 32. (in French)

[35] Congolese Institute for Nature Conservation. 2007. "The Biodiversity.” Accessed November 15, 2007. 
http://www.iccnrdc.cd/biodiversite.htm. (in French)

[36] Dowsett, R. J., Atkinson, P. W., and Caddick, J. A. 2015. "Checklist of the Birds of Democratic Republic of Congo." Accessed April 82015. http://www.africanbirdclub.org/sites/all/themes/abc_2/fpd f/testv11.php.

[37] Nigel, W. 1995. "Where to Watch Birds in Africa." Congo (formerly Zaire) Hotspots. Accessed November 14, 2007. http://www.camacdonald.com/birding/africacongo. htm.

[38] Lepage, D. 2015. "Avibase - Bird Checklists of the World Democratic Republic of Congo.” Accessed April 03, 2015. http://avibase.bsc-eoc.org/checklist.jsp?region $=$ cd\&list $=\mathrm{c}$ lements.

[39] Wikipedia. 2015. "List of Birds of the Democratic Republic of the Congo." Redirected from List of Birds of the Democratic Republic of Congo. Accessed April 02, 2015. http://en.wikipedia.org/wiki/List_of_birds_of_the Democratic_Republic_of_the_Congo.

[40] Wikipedia. 2015. "Birds Migration." Accessed April 1, 2015. http://fr.wikipedia.org/w/index.php?title=Migration des_oiseaux\&oldid=112870313.

[41] Punga. K. J. 2007. "Fauna of Humid Zone of the Democratic Republic of Congo." Nile Basin Initiative: Action Project of Nile Environmental Ttransfronariese, Workshop on Humid Zone and Biodiversity in the Democratic Republic of Congo, Handicapé Centre, Kinshasa, December 19-22, 2007. (unpublished) (in
French)

[42] Shumway, C., Musibono, D., Ifuta, S., Sullivan, J., Schelly, R., Punga, J., Palata, J. C., and. Puema, V. 2002. Biodiversity Survey: Systematics, Ecology and Conservation along the Congo River. Caribbean Renewable Energy Development Programme (CREDP), USAID.

[43] Punga, K. J. 2006. Rapport of Preliminary Participative Inventory in Communal Forest of Bobangi (Equator, Democratic Republic of Congo). Innovative Resources Management/USAID, 48. (unpublished) (in French)

[44] Bird Life International. 2008. "West African Raptors Are Faring Poorly Outside of Protected Areas." Bird Life State in the World's Birds Website. Accessed April 01, 2015. http://www.birdlife.org/datazone/sowb/casestudy/119.

[45] Bird Life International. 2009. "American Birds Sending Troubling Message about the Environment." Science Daily. Accessed April 01, 2015. www.sciencedaily.com/releases/2009/03/090323113103h tm.

[46] Frederiksen, M., Mavor, R. A., and Wanless, S. 2007. "Seabirds as Environmental Indicators: The Advantages of Combining Data Sets." Mar. Ecol. Prog. Ser. 352: 205-11.

[47] Mistry, J., Berardi, A., and Simpson, M. 2008. "Birds as Indicators of Wetland Status and Change in the North Rupununi, Guyana." Biodivrsity and Conservation 17 (10): 2383-409. 